
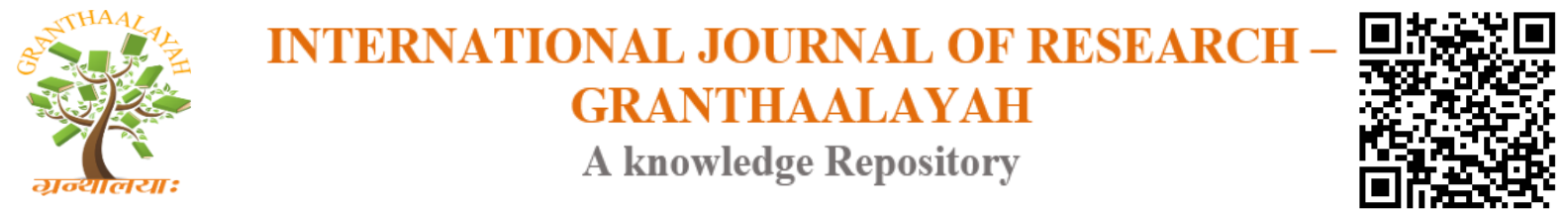

Science

\title{
TREATMENT OF SERVICE STATION WASTEWATER USING ELECTROCOAGULATION PROCESS
}

\author{
Dr. Krishna M. K ${ }^{1}$, Dr. Manjunath H.N², Ayesha Siddiqa ${ }^{3}$ \\ ${ }^{1}$ Professor, Department of Civil Engineering, S.J.B Institute of Technology, Bangalore -560060, \\ Karnataka, India \\ ${ }^{2}$ Associate Professor, Department of Civil Engineering, S.J.B Institute of Technology, \\ Bangalore -560060, Karnataka, India \\ ${ }^{3}$ Department of Civil Engineering, S.J.B Institute of Technology, Bangalore -560060, \\ Karnataka, India
}

\begin{abstract}
The various electrodes such as iron, aluminium, stainless steel and graphite are used in this study. Main objectives of the present paper is to evaluate the removal of COD, $\mathrm{pH}$, TDS and oil and grease from the automobile wastewater by Electro coagulation using iron as Monopolar configuration at different voltages and electrolysis time. To study the effect of inter electrode spacing and effect of different initial $\mathrm{pH}$ for the removal of selected parameter at constant voltage for the iron electrode. The removal efficiency decreases with increase in electrode spacing as $71.2 \%$ of COD, $96.5 \%$ of TDS and $88.76 \%$ of oil and grease removal was achieved with iron electrode.
\end{abstract}

Keywords: Electrocoagulation; Service Station Wastewater; Monopolar Configuration; Iron Electrode.

Cite This Article: Dr. Krishna M. K, Dr. Manjunath H.N, and Ayesha Siddiqa. (2017). "TREATMENT OF SERVICE STATION WASTEWATER USING ELECTROCOAGULATION PROCESS.” International Journal of Research - Granthaalayah, 5(7), 348-353. https://doi.org/10.29121/granthaalayah.v5.i7.2017.2140.

\section{Introduction}

Growth of urban population has increased the demand for fresh water sources and its rapid depletion has been a concern to ecologists. Rapid urbanization has led to hasty growth of service station in urban areas and this has necessitated the need to have automobile service centers at regular intervals. Service stations range from authorized service stations to small scale service centers, which undertake repair, washing and servicing of vehicles. According to the report provided by International Car Wash Association, a home car wash can go through 300 to 530 litres of water, whereas a wash at the garages will take about 115 to 170 liters and after the wash of vehicles, some water will also be used to wash floor and washing equipment's. This wash 
water contains paint, oil and grease, detergents, phosphates, hydrofluoric acid, ammonium bifluoride products and heavy metals. Fakhru'l-raziet.al. (2009) have given an overview of physical, chemical, biological and membrane treatment for waste water treatment. From the review it is reported that two or more methods may be used in series for effective water treatment and the selection of technology is decided based on the use of treated water. (MaliniAdapureddy, 2012)

Electrocoagulation is one of the commonly used processes for the treatment of wastewater, it involves the generation of coagulants in situ by dissolving the ions from the electrode. Electro means applying an electrical charge to water and coagulation means the process of reducing the colloidal particle surface charge and allowing suspended matter to form agglomeration. The various electrodes such as iron, aluminum, stainless steel and graphite are used in this technology. (Sekman, 2011) There has been renewed interest in the use of electro coagulation owing to the increase in environmental restrictions on wastewater. In the past decade, this technology has been increasingly used in developed countries for the treatment of industrial wastewaters, by allowing the particles to react with an ion having an opposite charge or floc of metallic hydroxides generated within the effluent. Electrocoagulation method is not only applied for the treatment of slaughterhouse wastewater, wastewater containing heavy metals, pesticides and phenol compounds, drinking water for fluoride removal, but also for the treatment of automobile wastewater.

The currently available conventional treatment technologies for the treatment of automobile service station wastewater consist of a series of physical, chemical and biological methods. But some soluble organic compound goes untreated in these treatment steps, resulting in increased levels of pollutants in the treatment plant effluents. However, there is no single technology that can meet all requirements according to the variable nature of the wastewater. Electro coagulation process is playing a more prominent role in the treatment of automobile service station wastewater by virtue of various benefits including environmental compatibility, versatility, energy efficiency, safety, selectivity, amenability to automation and cost effectiveness.( krishna.M.K-2017)

\section{Materials and Methodology}

\subsection{Experimental Setup}

Experiments were carried out as batch mode in 2 liter capacity circular borosil glass beaker, for Monopolar configuration of electro coagulation technique. Fig 1 shows the schematic diagram of experimental set up. The iron electrode with dimension $5 \mathrm{~cm}$ X $5 \mathrm{~cm}$ and $1 \mathrm{~mm}$ thickness were used in this present study. Experiments were carried with different voltages, varying inter electrode spacing. For each voltage at every 30 minutes intervals samples are taken and analyzed for $\mathrm{pH}, \mathrm{COD}$, TDS, oil and grease removal efficiency is studied for iron electrode. The electric power required during the electrolysis was provided by a laboratory Dual DC power supply unit $(0-30 \mathrm{~V}, 2 \mathrm{~A})$.Copper wires were connected to the electrodes to supply the DC current to the system. The wastewater for this study is collected from various automobile service stations, the collected wastewater was analyzed as per standard methods. 


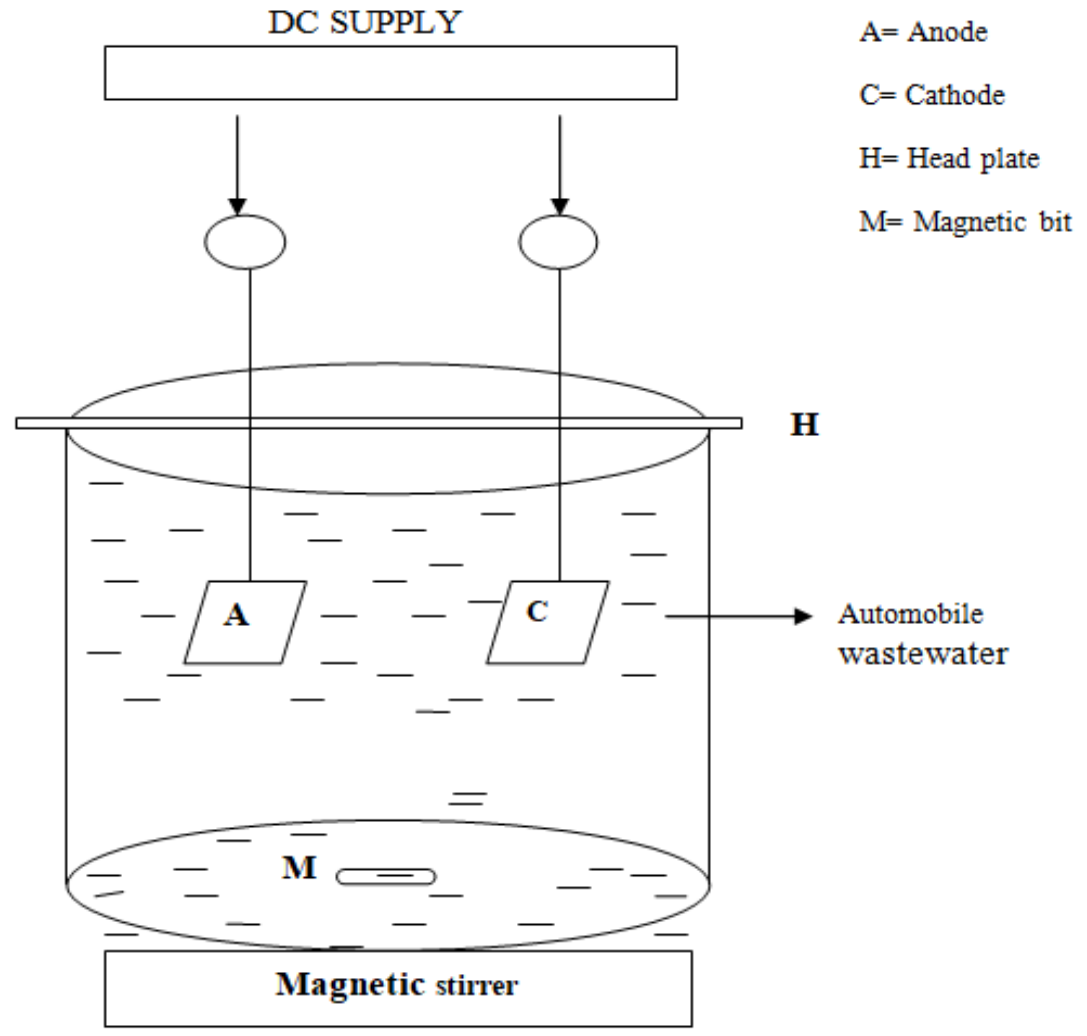

DOI: 10.5281/zenodo.837276

Figure 1: Schematic diagram of electro coagulation unit

\subsection{Methodology for Different Electrode Spacing, Electrolysis Time and Voltage}

Monopolar configuration of two iron plate electrodes was used for electro coagulation. Iron electrodes are placed at inter electrode gap of $1 \mathrm{~cm}$ up to $3 \mathrm{~cm}$ at an interval of $1 \mathrm{~cm}$, on the fiber electrode holding stand fixed to the mouth of the reactor and connected to the $30 \mathrm{~V}$ dual DC power supply. The batch experiment was conducted at varying voltage of $10 \mathrm{~V}, 20 \mathrm{~V}$ and $30 \mathrm{~V}$ with selected time intervals of 30,60, and 90 up to 180 minutes at room temperature. In each run, approximately two liters of automobile wastewater sample was placed in reactor.

\section{Results and Discussion}

Performance evaluation of electro coagulation process with the iron electrodes was carried out for different voltages of $10 \mathrm{~V}, 20 \mathrm{~V}, 30 \mathrm{~V}$ with selected times of 30 to 180 minutes at time intervals 30 minutes. Different electrode spacing of $1 \mathrm{~cm}$ up to $3 \mathrm{~cm}$ at an interval of $1 \mathrm{~cm}$ was discussed. Removal efficiency for all the operating parameters was discussed and presented.

\subsection{Characteristics of Automobile Wastewater}

In the present study wastewater collected from automobile service stations was analyzed as per standard methods and presented in Table 1. 
Table 1: Characteristics of automobile wastewater

\begin{tabular}{|l|l|}
\hline Parameter & Values \\
\hline Acidity $\mathrm{mg} / \mathrm{l}$ & 84 \\
\hline $\mathrm{pH}$ & 7.83 \\
\hline COD mg/L & 348 \\
\hline Alkalinity mg/l & 404 \\
\hline TDS mg/L & 400 \\
\hline Oil and grease mg/L & 450 \\
\hline Chlorides mg/l & 134 \\
\hline Total hardness mg/l & 260 \\
\hline BOD mg/l & 69.5 \\
\hline
\end{tabular}

\subsection{Effect of Electrical Potential and Electrolysis Time}

The results and percentage removal efficiency for varying electrical potential of $10 \mathrm{~V}, 20 \mathrm{~V}$ and $30 \mathrm{~V}$, electrolysis time of 30, 60 up to 180 minutes at electrode spacing of $2 \mathrm{~cm}$ and iron electrode Figure 2-4. Figure 2 depicts the COD removal efficiency at different voltages and time intervals

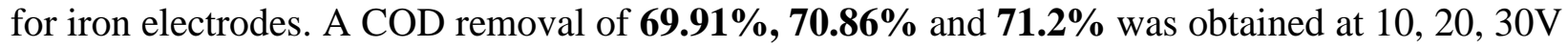
respectively and optimum removal of COD 71.2\% was achieved at 30V for contact time of 180 min.COD removal efficiency increases as the electrical potential and electrolysis time increases. This is due to the release of metal ions increases with increase in electrical potential. Therefore, there is an increase in floc formation and hence an enhancement in the COD removal efficiency.

Figure 3 illustrates TDS removal efficiency at different voltages and time intervals for iron electrodes. About 92.98\%, 94.18\% and 96.5\% was achieved at 10, 20,30V respectively and an optimum removal of $\mathbf{9 6 . 5 \%}$ was achieved at $30 \mathrm{~V}$ for contact time of 90 minutes and after that it remains constant up to 180 minutes. The hydrogen gas liberated at the cathode helps to float the contaminants this influences the removal of TDS. The TDS removal efficiency increases with increase in electrical potential and electrolysis time.

The effect of voltage and electrolysis time on oil and grease removal is shown in the Figure 4. Optimum removal of $88.76 \%$ was observed at $30 \mathrm{~V}$ and contact time of 180 minutes after that increasing electrolysis time did not affect the removal rate significantly. Increase in voltage enhances the generation of hydrogen and oxygen gases formed at electrode surfaces. This leads to an increase in the number of gas bubbles inside the EC unit Consequently the attachment step between gas bubbles and oil drop is enhanced and more oil drops are carried by gas bubbles to the surface and oil removal was increased.

From the figures it was concluded that increase in voltage and contact time increases the removal efficiency and optimum removal efficiency with the iron electrode is achieved at $30 \mathrm{~V}$ and contact time of 180 minutes for COD, TDS and oil and grease. Hence the optimum voltage is $30 \mathrm{~V}$ and 180 minutes electrolysis time. Gharemani et al.2012stated that density of bubbles increases and their size decreases with an increase in electrical potential results in a greater faster and further removal of pollutants. A greater amount of hydroxide flocs was produced by increasing the electric current causing an efficient as per Faraday's law of electrolysis which 
states that the mass of a substance deposited at an electrode during electrolysis is directly proportional to the quantity of charge passed through the electrolyte. As a result increasing the contact time in the electro coagulation process gives rise to a higher concentration of free ions inside the system and consequently removal efficiency was increased.

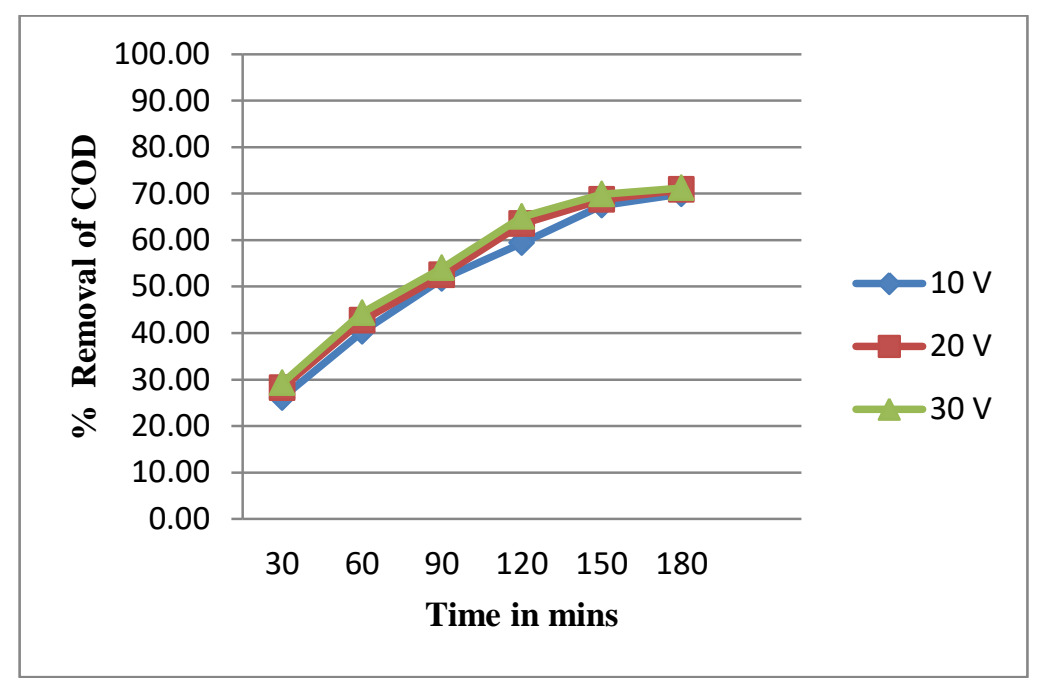

Figure 2: COD removal efficiency at different voltage and time

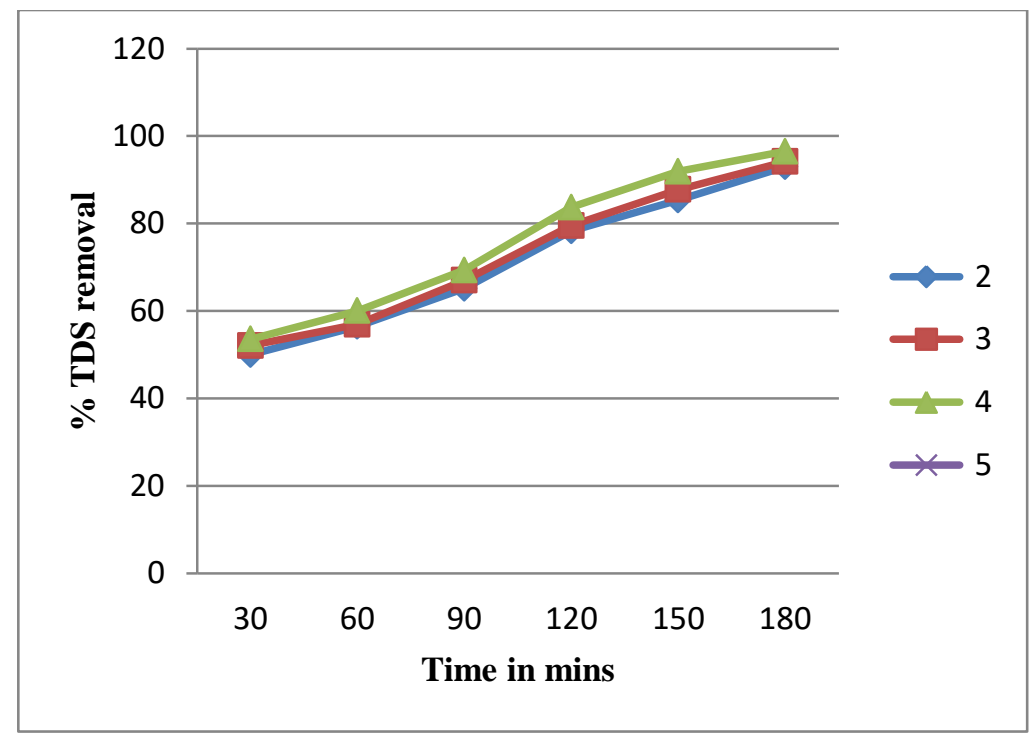

Figure 3: TDS removal efficiency at different voltage and time 


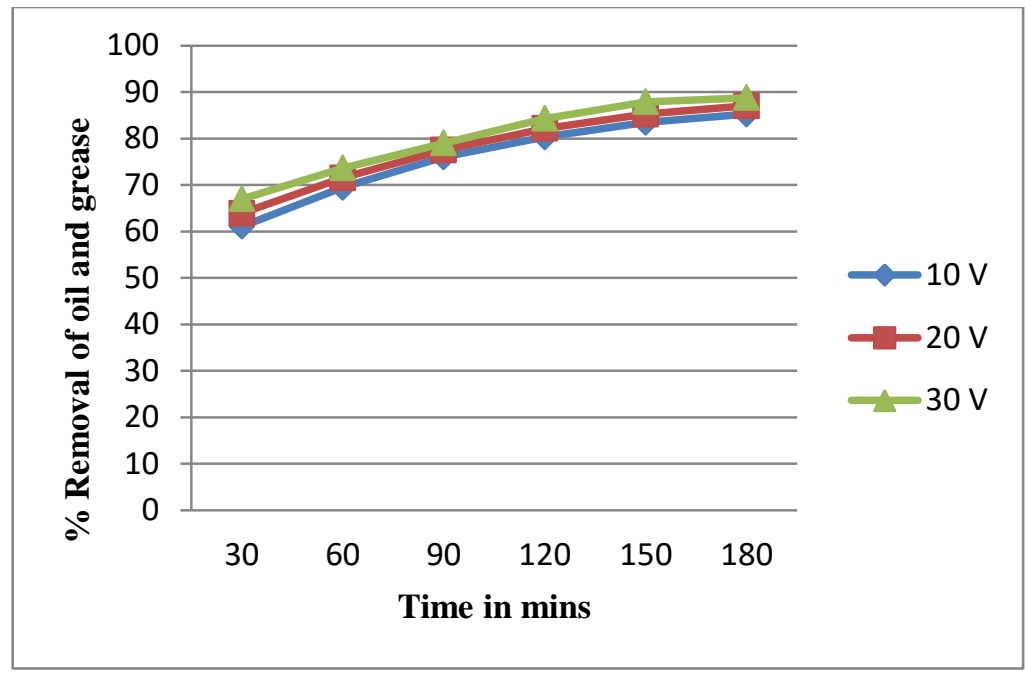

Figure 4: Oil and grease removal efficiency at different voltage and time

\section{Conclusions}

Based on the experimental results obtained from batch studies following conclusions have been drawn.

- The increase in voltage and electrolysis time increases the removal efficiency.

- The removal efficiency of COD, TDS, and oil and grease were $71.2 \%, 96.5 \%, 88.76 \%$ respectively under the optimum voltage and electrolysis time was $30 \mathrm{~V}$ and 180 minutes for iron electrodes.

- The iron electrode shows more removal efficiency for COD, TDS and oil and grease.

- Hence automobile wastewater can be treating efficiently with the iron electrode under all the operational conditions.

\section{References}

[1] Krishna.M.K , CSMV Prasad "Feasibility study of Dairy Wastewater by Electrocoagulation Technique"proceedings of the 49th Indian water works association held at Nagpur 19-21 jan (2017) pp-67.

[2] Kashefialas.M, Khosrav.M, Marandi.R and Seyyedi.K. "Treatment of dye solution containing colored index acid yellow 36 by electrocoagulation using iron electrodes" .International journal of Environmental science and technology Vol. 2, No. 4, pp. 365-371(2006 ).

[3] Ghahremani N, Bagheri S, Hassani S.M, Khoshchehreh M.R. "Treatment of Dairy Industry Wastewater using an Electrocoagulation Process", Advances in environmental biology, Vol 6(7), pp 1897-1901(2012)

[4] Sekman, E., Top, S., Uslu, E., Varank, G. and Bilgili, M. S. "Treatment of Oily Wastewater from Port Waste Reception Facilities by Electrocoagulation". International journal of Environ. Res., 5(4):1079- 1086(2011).

[5] MaliniAdapureddy and SudhaGoel, "Optimizing Electrocoagulation of drinking water for Turbidity removal in a batch reactor", International conference on Environmental and Technology", vol 30(2012). 